\title{
Suplementação nutracêutica e fitoterápica no tratamento da fibromialgia: revisão de
}

\section{literatura}

\author{
Nutraceutical and phytotherapy supplementation in the treatment of fibromyalgia: literature \\ review
}

Suplementación nutracéutica y fitoterapéutica en el tratamiento de la fibromialgia: revisión de la

literatura

Recebido: 26/01/2021 | Revisado: 02/02/2021 | Aceito: 29/10/2021 | Publicado: 31/10/2021

\author{
Jefferson Jovelino Amaral dos Santos \\ ORCID: https://orcid.org/0000-0003-2204-631X \\ Universidade Paranaense, Brasil \\ E-mail: jeffe_fisio@hotmail.com.br \\ Ana Paula Tardivo \\ ORCID: https://orcid.org/0000-0002-1716-1041 \\ Centro Universitário Filadélfia, Brasil \\ E-mail: posortolondrina@hotmail.com \\ Thaís Cristina Coelho de Ornelas Salasar \\ ORCID: https://orcid.org/0000-0001-5813-6187 \\ Centro Universitário Filadélfia, Brasil \\ E-mail: posortolondrina@ hotmail.com \\ Victor Nascimento dos Santos \\ ORCID: https://orcid.org/ 0000-0001-5161-5425 \\ Universidade Estadual do Oeste do Paraná, Brasil \\ E-mail: reabplus@yahoo.com.br
}

\begin{abstract}
Resumo
A Fibromialgia (FB) é uma doença multifatorial que atinge uma grande parcela da população e ainda tem o seu diagnóstico como motivo de controvérsia. É caracterizada pela dor crônica em múltiplos pontos do corpo. O presente artigo teve como objetivo identificar ativos nutracêuticos que pudessem contribuir ao tratamento da FB nos seus múltiplos aspectos e assim, melhorar a qualidade de vida de seus portadores. O levantamento bibliográfico delimitouse na busca de artigos em português e inglês nas bases de dados PubMed, SciELO e LILAC, publicados no período de janeiro de 2015 a março de 2020. Os princípios ativos mais encontrados no tratamento da FB foram a coenzima Q10, 25-hidroxivitamina D, probióticos (Lactobacillus Rhamnosus, Casei, Acidophilus e Bifidobacterium Bifidus) e fitoterápicos como gengibre, acerola, Spiraea Ulmari, passiflora, Elymus repens, em associação com outros produtos, o Crocus Sativus (açafrão) além do Ganoderma lucidum que é um tipo de cogumelo. É de fundamental importância a compreensão de como os suplementos podem auxiliar no tratamento da FB promovendo uma terapêutica mais natural, menos dispendiosa e com menos efeitos colaterais para os seus portadores.
\end{abstract}

Palavras-chave: Fibromialgia; Suplemento dietético; Nutracêutico; Estresse oxidativo.

\begin{abstract}
Fibromyalgia (FB) is a multifactorial disease that affects a large portion of the population and still has its diagnosis as a subject of controversy. It is characterized by chronic pain in multiple points of the body. This article aimed to identify nutraceutical supplements that could contribute to the treatment of FB in its multiple aspects and thus improve the quality of life of the patients. The bibliographic survey was limited to searches for articles in Portuguese and English in the PubMed, SciELO and LILACS databases published between January 2015 and March 2020. The most commonly found active ingredients were coenzyme Q10, 25-hydroxyvitamin D, probiotics (Lactobacillus Rhamnosus, Casei, Acidophilus and Bifidobacterium Bifidus) and phytotherapic medicines such as ginger, acerola cherry, Spiraea Ulmaria, passiflora, Elymus repens in association with other products, Crocus Sativus (saffron) and also Ganoderma lucidum, a kind of mushroom. It is of fundamental importance to understand how supplements can help in the treatment of fibromyalgia, promoting a more natural therapy, less expensive and with less side effects for its patients.
\end{abstract}

Keywords: Fibromyalgia; Dietary supplement; Nutraceutical; Oxidative stress.

\section{Resumen}

La fibromialgia (FB) es una enfermedad multifactorial que afecta a una gran parte de la población y aún tiene su diagnóstico como tema de controversia. Se caracteriza por dolor crónico en múltiples puntos del cuerpo. Este artículo 
tuvo como objetivo identificar activos nutracéuticos que pudieran contribuir al tratamiento de la FB en sus múltiples aspectos y, así, mejorar la calidad de vida de sus pacientes. El relevamiento bibliográfico fue limitado en la búsqueda de artículos en portugués e inglés en las bases de datos PubMed, SciELO y LILAC, publicadas entre enero de 2015 y marzo de 2020. Los principios activos más comunes encontrados en el tratamiento de la FB fueron la coenzima Q10, 25-hidroxivitamina D, probióticos (Lactobacillus Rhamnosus, Casei, Acidophilus y Bifidobacterium Bifidus) y medicinas a base de hierbas como jengibre, acerola, Spiraea Ulmari, pasiflora, Elymus repens, en asociación con otros productos, Crocus Sativus (azafrán) y un tipo de hongo Ganoderma. Es de fundamental importancia entender cómo los suplementos pueden ayudar en el tratamiento de la LM, promoviendo una terapia más natural, menos costosa y con menos efectos secundarios para sus pacientes.

Palabras clave: Fibromialgia; Suplemento dietético; Nutracéutico; Estrés oxidativo.

\section{Introdução}

A Fibromialgia (FB) é uma doença comum, porém contestada. É definida como uma disfunção que apresenta múltiplos pontos sensíveis (avaliados durante o exame físico) e dor crônica generalizada (Wolfe et al., 2010).

Trata-se de um distúrbio comum, que ocorre em todas as populações do mundo. Em geral, a prevalência de pacientes com sintomas que atendem aos critérios de diagnóstico está entre $2 \%$ e $4 \%$ na maioria dos estudos atuais tendo como causa fatores genéticos e biológicos que podem ser amplificados por aspectos psicológicos, comportamentais e sociais incluindo depressão, ansiedade, transtorno obsessivo-compulsivo e transtorno de estresse pós-traumático. Os neurotransmissores que mediam a transmissão da dor também podem afetar o humor, a memória, a fadiga e o sono (Häuser et al., 2015).

Os mecanismos fisiopatológicos ligados a FB podem ser resumidos em: fatores genéticos, processos neurais, neuroinflamação e estresse oxidativo e, a partir de tais mecanismos, os tratamentos mais propostos são os recursos não farmacológicos (terapia cognitiva não comportamental, exercício físico, Qigong, tai-chi, ioga e educação do paciente); recursos farmacológicos (amitriptilina, ciclobenzaprina, duloxetina e pregabalina) e recursos complementares (CoQ10, L-Carnitina e Luteolina)(Theoharides et al., 2015).

O presente estudo teve como objetivo destacar a terapia complementar baseada na suplementação nutracêutica e fitoterápica para FB evidenciada pela pesquisa científica fundamentada por critérios rígidos de estudo.

\section{Metodologia}

O presente estudo trata-se de uma revisão de literatura, por meio de método qualitativo (Pereira et al., 2018), a qual teve como objetivo a catalogação e análise de pesquisas relevantes, fornecendo base para tomada de decisão na prática clínica das profissões habilitadas para a prescrição de suplementação nutracêutica, além de levantar pontos fracos no estado da arte deste tipo de tratamento para a realização de novos estudos.

A busca dos artigos científicos foi realizada por meio das bases de dados PubMed (National Center for Biotechnology Information - NCBI, U.S. National Library of Medicine), SciELO (Scientific Eletronic Library Online) e LILACS (Literatura Latino-Americano e do Caribe em Ciências da Saúde). Foram utilizadas as seguintes palavras-chave encontradas nos DeCS Descritores em Ciências da Saúde: "FB e suplementos dietéticos", "fibromyalgia and Dietary Supplements", "FB e nutracêuticos", "fibromyalgia and nutraceutical", "FB e terapia ortomolecular", "fibromyalgia and Orthomolecular Therapy", "FB e estresse oxidativo" e "fibromyalgia and oxidative stress".

A pesquisa nas bases de dados foi realizada a partir de estudos publicados no período entre janeiro de 2015 a março de 2020, sendo a seleção inicial realizada apenas com base nos títulos dos artigos. Os resumos dos estudos selecionados foram lidos e avaliados de acordo com os critérios de inclusão e exclusão.

Foram definidos como critérios de inclusão: estudos clínicos; publicações dos últimos cinco anos; nos idiomas português e inglês; que abordassem a relação existente entre a suplementação dietética e ortomolecular para FB, considerando os aspectos de dor, limitação funcional e estado mental destes pacientes. 
Foram definidos como critérios de exclusão: revisões integrativas, sistemáticas e meta-análises; trabalhos que não apresentassem resumos na íntegra; artigos incompletos; resumos de congressos; trabalhos repetidos e que não se relacionavam aos assuntos considerados.

Todos os artigos revisados foram aprovados em Comitês de Ética de Pesquisa em Seres Humanos de suas instituições. Este estudo baseou-se na Declaração dos Relatórios Preferenciais para Revisão Sistemática PRISMA (Liberati et al., 2009) (Figura 1).

Figura 1 - Fluxograma de Identificação e seleção de artigos.

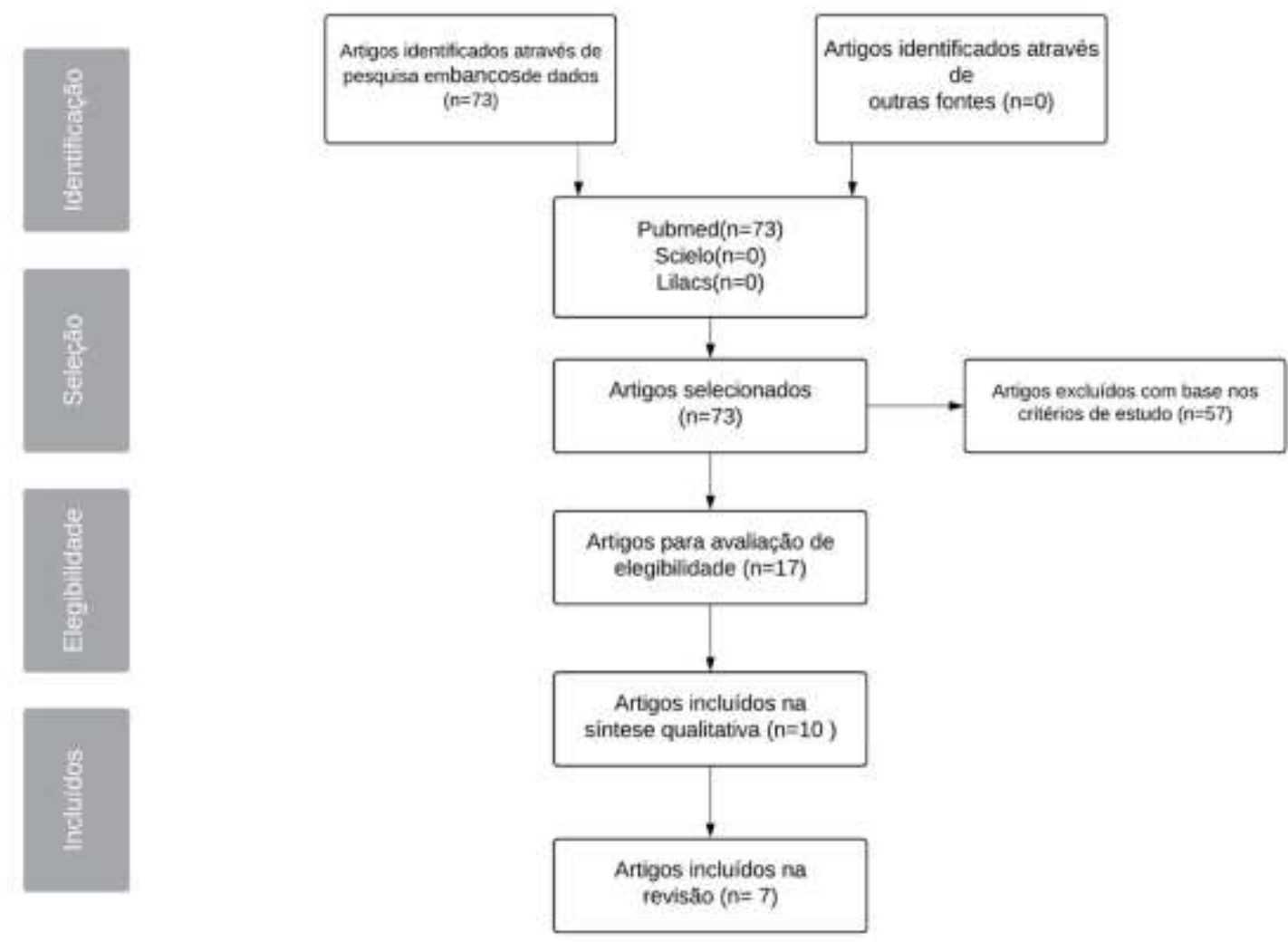

Fonte: Dados da pesquisa.

A discussão foi categorizada em 4 temáticas principais as quais se relacionam aos ativos mais encontrados: Coenzima Q10 (COQ10), 25-hidroxivitamina D, probióticos e fitoterápicos.

\section{Resultados}

Com a utilização dos descritores citados anteriormente foi possível encontrar 73 artigos na base de dados Pubmed e nenhum artigo nas demais bases (Figura 1) no intervalo de tempo especificado. Destes 73 após submissão aos critérios de elegibilidade e síntese qualitativa somente seis puderam ser utilizados na presente pesquisa.

Embora nos últimos anos vários estudos tenham sido publicados, a FB ainda tem sido sub, super ou mal diagnosticada 
atualmente (Hauser, Sarzi-Puttini, \& Fitzcharles, 2019). Nota-se que é crescente o consenso nas pesquisas que uma abordagem multidisciplinar pode contribuir para a melhora do quadro e da qualidade de vida dos portadores desta disfunção (BinkiewiczGlinska et al., 2015).

O manejo multidisciplinar do paciente portador de FB através de uma abordagem holística e com utilização de técnicas mais naturais poderia evitar sua supermedicação e também diminuir os custos com terapias caras ou que causem dependência ao usuário.

No Quadro 1 demonstra-se um resumo dos sete artigos de maior relevância encontrados e que cumpriram os critérios de seleção.

Quadro 1: Sinopse dos artigos incluídos na revisão.

\begin{tabular}{|c|c|c|c|c|c|}
\hline Autor, Ano. & Indexado & População & Objetivo do estudo & Metodologia do Estudo & Desfecho do estudo \\
\hline $\begin{array}{l}\text { Collado Mateo } \\
\text { et al. } \\
(2015)(\text { Collado } \\
\text { Mateo et al., } \\
\text { 2015) }\end{array}$ & Pubmed & 64 pacientes & $\begin{array}{l}\text { Avaliar os efeitos sobre a condição } \\
\text { física em mulheres com FB } \\
\text { submetidas a um tratamento de seis } \\
\text { semanas com Ganoderma lucidum e } \\
\text { comparar com os tratamentos com } \\
\text { ceratonia siliqua. }\end{array}$ & $\begin{array}{l}\text { As pacientes utilizaram } 6 \mathrm{~g} \text { de } \\
\text { Ganoderma lucidum ou Ceratonia } \\
\text { siliqua por dia durante } 6 \text { semanas. } \\
\text { Diferentes testes de aptidão foram } \\
\text { selecionados para avaliar a capacidade } \\
\text { funcional. }\end{array}$ & $\begin{array}{l}\text { O Ganoderma lucidum melhorou } \\
\text { significativamente a resistência } \\
\text { aeróbica, a flexibilidade do corpo } \\
\text { e a velocidade ( } p<0,05) \text {. Não } \\
\text { houve melhora significativa em } \\
\text { nenhum teste físico no grupo } \\
\text { Ceratonia siliqua. }\end{array}$ \\
\hline $\begin{array}{l}\text { Di Pierro et al } \\
\text { (2017)(Di } \\
\text { Pierro, Rossi, } \\
\text { Consensi, } \\
\text { Giacomelli, \& } \\
\text { Bazzichi, 2017) }\end{array}$ & Pubmed & 22 pacientes & $\begin{array}{l}\text { Analisar o efeito da administração de } \\
\text { uma forma solúvel em água de CoQ10 } \\
\text { em mulheres com diagnóstico de FB. }\end{array}$ & $\begin{array}{l}\text { Dois grupos aleatórios foram tratados } \\
\text { por } 3 \text { meses com uma fórmula à base } \\
\text { de CoQ10 solúvel em água (Grupo } \\
\text { tratado) ou como controle, com um } \\
\text { suplemento isento de CoQ10 } \\
\text { comparável. Após } 3 \text { meses, os dois } \\
\text { grupos foram revertidos. Os } \\
\text { tratamentos foram administrados duas } \\
\text { vezes ao dia. }\end{array}$ & $\begin{array}{l}\text { Efeitos benéficos exercidos pela } \\
\text { CoQ10 contra a dor, fadiga, } \\
\text { distúrbios do sono e dificuldades } \\
\text { mentais em mulheres afetadas por } \\
\text { FB. }\end{array}$ \\
\hline $\begin{array}{l}\text { De Carvalho et } \\
\text { al (2018)(de } \\
\text { Carvalho, da } \\
\text { Rocha Araujo, } \\
\text { da Mota, Aires, } \\
\text { \& de Araujo, } \\
\text { 2018) }\end{array}$ & Pubmed & 11 pacientes & $\begin{array}{l}\text { Analisar os efeitos de } 3 \text { meses de } \\
\text { suplementação } \quad \text { com }\end{array}$ & $\begin{array}{l}\text { Avaliar o nível de dor pela Escala } \\
\text { Visual Analógica de Dor (EVA) e } \\
\text { pelo número de Tender Points } \\
\text { encontrados. }\end{array}$ & $\begin{array}{l}\text { Diminuição no nível de dor após } \\
\text { o uso da } \\
\text { 25-Hidroxivitamina D. }\end{array}$ \\
\hline $\begin{array}{l}\text { Roman et al. } \\
\text { (2018)(Pablo } \\
\text { Roman et al., } \\
\quad \text { 2018) }\end{array}$ & Pubmed & 40 pacientes & $\begin{array}{l}\text { Testar se um probiótico multiespécie } \\
\text { (Lactobacillus Rhamnosus GG®, } \\
\text { Casei, Acidophilus, e Bifidobacterium } \\
\text { Bifidus.) poderia melhorar a cognição, } \\
\text { sintomas emocionais e estado } \\
\text { funcional em uma população } \\
\text { diagnosticada com FB. }\end{array}$ & $\begin{array}{l}\text { Estudo piloto de intervenção duplo } \\
\text { cego randomizado controlado, com } \\
\text { um grupo controle placebo e um } \\
\text { grupo experimental. Dor, impacto da } \\
\text { FB na qualidade de vida, ansiedade e } \\
\text { sintomas depressivos foram medidos } \\
\text { durante as fases pré e pós-intervenção; } \\
\text { os participantes também concluíram } \\
\text { duas tarefas cognitivas } \\
\text { computadorizadas para avaliar a } \\
\text { escolha impulsiva e a tomada de } \\
\text { decisões e a concentração urinária de } \\
\text { cortisol. }\end{array}$ & $\begin{array}{l}\text { Os resultados indicaram que os } \\
\text { probióticos melhoraram a } \\
\text { impulsividade e a tomada de } \\
\text { decisão nesses pacientes. }\end{array}$ \\
\hline $\begin{array}{l}\text { Shakiba et al. } \\
\text { (2018)(Shakiba } \\
\text { et al., 2018) }\end{array}$ & Pubmed & 46 pacientes & $\begin{array}{llr}\text { Comparar a eficácia do Crocus } \\
\text { Sativus com a duloxetina em } \\
\text { pacientes com FB. }\end{array}$ & $\begin{array}{l}\text { Estudo duplo-cego randomizado com } \\
\text { grupo controle onde os grupos } \\
\text { receberam } 15 \mathrm{mg} \text { de Crocus Sativus } \\
\text { ou } 30 \mathrm{mg} \text { de duloxetina iniciando com } \\
\text { uma capsula ao dia na primeira } \\
\text { semana e duas cápsulas ao dia após a } \\
\text { segunda semana numa duração total } \\
\text { de } 8 \text { semanas. }\end{array}$ & $\begin{array}{lrr}\text { O Crocus } & \text { Sativus demonstrou } \\
\text { eficácia } & \text { comparável no } \\
\text { tratamento da FB. } & \end{array}$ \\
\hline $\begin{array}{l}\text { Sawaddiruk et } \\
\text { al. } \\
(2019)(\text { Sawaddi } \\
\text { ruk et al., 2019) }\end{array}$ & Pubmed & 11 pacientes & $\begin{array}{l}\text { Verificar se a suplementação de } \\
\text { CoQ10 com pregabalina reduziria o } \\
\text { escore de dor e ansiedade melhor do } \\
\text { que a pregabalina sozinha, via } \\
\text { redução da atividade cerebral, estresse }\end{array}$ & $\begin{array}{l}\text { Estudo randomizado, duplo-cego, } \\
\text { controlado por placebo. Onze } \\
\text { pacientes com FB foram incluídos } \\
\text { com } 2 \text { semanas de lavagem e alocados } \\
\text { aleatoriamente em } 2 \text { grupos de }\end{array}$ & $\begin{array}{l}\text { A pregabalina sozinha reduziu a } \\
\text { dor e a ansiedade através da } \\
\text { diminuição da atividade cerebral } \\
\text { em comparação com a linha de } \\
\text { base. No entanto, não afetou o }\end{array}$ \\
\hline
\end{tabular}




\begin{tabular}{|c|c|c|c|c|c|}
\hline & & & $\begin{array}{l}\text { oxidativo mitocondrial, aumento dos } \\
\text { níveis de antioxidantes e diminuição } \\
\text { da inflamação em pacientes com FM. }\end{array}$ & $\begin{array}{l}\text { tratamento; pregabalina com CoQ10 } \\
\text { ou pregabalina com placebo por } 40 \\
\text { dias. Em seguida, os pacientes do } \\
\text { grupo CoQ10 foram trocados para } \\
\text { placebo e os pacientes do grupo } \\
\text { placebo foram transferidos para } \\
\text { CoQ10 por mais } 40 \text { dias. O limiar de } \\
\text { pressão da dor, questionário de FB, } \\
\text { ansiedade e escore de dor foram } \\
\text { administrados. As células } \\
\text { mononucleares do sangue periférico } \\
\text { foram isoladas para investigar o } \\
\text { estresse oxidativo mitocondrial a a } \\
\text { inflamação nos dias } 0,40 \text { e } 80\end{array}$ & $\begin{array}{l}\text { estresse oxidativo mitocondrial e } \\
\text { a inflamação. A suplementação } \\
\text { com CoQ10 reduziu mais } \\
\text { efetivamente a dor, ansiedade e } \\
\text { atividade cerebral, estresse } \\
\text { oxidativo mitocondrial e } \\
\text { inflamação. A CoQ10 também } \\
\text { aumentou os níveis reduzidos de } \\
\text { glutationa e os níveis de } \\
\text { superóxido dismutase (SOD) em } \\
\text { pacientes com FM }\end{array}$ \\
\hline $\begin{array}{l}\text { Barmaki et al. } \\
\text { (2019)(Barmaki } \\
\text { et al., 2019) }\end{array}$ & Pubmed & 100 pacientes & $\begin{array}{l}\text { Avaliar a eficácia e a segurança de um } \\
\text { tratamento fitoterápico (Fib-19-01) ao } \\
\text { atual regime terapêutico em pacientes } \\
\text { com FM. } \\
\text { Ponto final primário: alteração na FB } \\
\text { entre o dia } 0 \text { e o mês } 6 \text { (M6). Critérios } \\
\text { Secundários: variação ao longo do } \\
\text { tempo na FB, alteração na escala de } \\
\text { fadiga, qualidade do sono, qualidade } \\
\text { de vida e Ansiedade e depressão. }\end{array}$ & $\begin{array}{l}\text { Estudo controlado } r \text { duplo-cego: } \\
\text { mulheres com FB ativa foram } \\
\text { randomizadas para receber Fib-19-01 } \\
\text { ou um suplemento alimentar (FS) } \\
\text { indistinguível de Fib-19-01 ou sem } \\
\text { tratamento suplementar (NoST). } \\
\text { Todos continuaram a terapia } \\
\text { convencional ao longo dos } 6 \text { meses de } \\
\text { acompanhamento. }\end{array}$ & $\begin{array}{l}\text { Em pacientes com FB em uso de } \\
\text { terapia convencional, a Fib-19-01 } \\
\text { tem um efeito terapêutico na } \\
\text { fadiga, emoção e vida social e } \\
\text { depressão associada à doença. }\end{array}$ \\
\hline
\end{tabular}

Fonte: Dados da pesquisa.

\section{Discussão}

A compreensão atual da FB concentra-se principalmente na percepção neuronal alterada, resultando em sensibilidade generalizada a vários estímulos devido a alterações no sistema nervoso central, neuroendócrino e nervoso. Vários processos neuroinflamatórios e neurogênicos podem contribuir para os sintomas da FB. Com base nesses mecanismos essenciais de hipersensibilidade generalizada, a propriocepção aumentada dos segmentos osteomusculares geralmente ocorre em um padrão de distribuição da dor axial-simétrica (bilateral). O processamento relacionado ao estresse alterado provavelmente explica a alta prevalência de sintomas vegetativos, afetivos e cognitivos (psicológicos) comórbidos na FB e que níveis mais altos de estresse estão associados a limiares de dor à pressão reduzidos. Consequentemente, a hiperalgesia induzida por estresse a longo prazo foi relacionada como fator fisiopatológico crucial entre a exposição crônica ao estresse e a sensibilização generalizada à dor (Stewart et al., 2019).

\section{Coenzima Q 10 (CoQ10)}

A CoQ10 ou Ubiquinona é uma substância presente em todas as membranas de todas as células do corpo que transfere elétrons dos complexos I e II para o complexo III na cadeia respiratória mitocondrial e cumpre um papel essencial na produção de ATP mitocondrial, influenciando o metabolismo celular; regulação de proteínas de desacoplamento, poro de transição, $\beta$ oxidação de ácidos graxos e via nucleotídica; e também limitando a produção de espécies reativas de oxigênio (estresse oxidativo)(Cordero et al., 2011).

Estudos demonstraram que o estresse oxidativo é presente na FB(Cordero et al., 2010) e que a CoQ10 pode exercer um papel fundamental neste quadro, melhorando a função mitocondrial e, consequentemente, o metabolismo celular(Cordero et al., 2011).

A presente pesquisa além dos efeitos relatados anteriormente pôde verificar que a CoQ10 associada a pregabalina reduziu mais efetivamente a dor, ansiedade e atividade cerebral, estresse oxidativo mitocondrial e inflamação do que a administração isolada da pregabalina. A CoQ10 também aumentou os níveis reduzidos de glutationa e os níveis de superóxido dismutase (SOD)(Sawaddiruk et al., 2019). 
Os efeitos antioxidantes da CoQ10 tem sido demonstrados em diversos trabalhos nas mais variadas disfunções nas quais o estresse oxidativo esteja presente (Mizobuti et al., 2019), (Jorat et al., 2019),(Mousavinejad et al., 2018),(Ramezani, Sahraei, Simani, Heydari, \& Shahidi, 2018). Os pacientes portadores de FB podem se beneficiar do uso da CoQ10 em doses usuais de 300mg ao dia (Cordero et al., 2011).

Em suma, com base nos princípios fisiológicos discutidos, o uso da CoQ10 pode produzir efeitos positivos em paciente com FB no controle da dor, distúrbios do sono e dificuldades mentais (Di Pierro et al., 2017).

\section{5-Hidroxivitamina D}

A vitamina D é sintetizada na pele após exposição solar e sua síntese endógena de vitamina D é de $80 \%$ das suas necessidades diárias necessárias. O restante provém de fontes alimentares vegetais e animais (oleosas, peixe, gema de ovo, leite fortificado) como ergocalciferol (Vitamina D2) e colecalciferol (vitamina D3), respectivamente (Martins, Cardinali, Ravanelli, \& Brunaldi, 2020).

A atrofia muscular, particularmente de fibras do tipo II, tem sido descrita histopatologicamente na deficiência de vitamina $\mathrm{D}$ cujos metabólitos afetam o metabolismo das células musculares por três vias principais: a) mediando a transcrição de genes; b) por vias rápidas que não envolvem síntese de DNA e c) pela variante alélica do receptor de vitamina D (VDR) (Sahota, 2014).

Pode-se encontrar VDRs em células musculares esqueléticas que se ligam especificamente a 1,25 (OH) 2D. A suplementação de vitamina $\mathrm{D}$ induz mudanças rápidas no metabolismo das células musculares, que não podem ser explicadas por um lento caminho genético. Isso é possível através da ação do VDR atuando diretamente na membrana da célula muscular. Na ligação, várias vias secundárias do mensageiro são ativadas, resultando em aumento da captação de cálcio (em minutos), tanto pelos canais de cálcio dependentes da voltagem quanto pelos canais de cálcio ativados pela liberação de cálcio. A força muscular também parece ser influenciada pelo genótipo do VDR na célula muscular e com o uso de endonucleases de restrição específicas (Sahota, 2014).

Estudos recentes demonstram que não há consenso sobre a relação entre $\mathrm{FB}$ e vitamina $\mathrm{D}$, em particular a relação causa-efeito (Ellis, Kelly, Shurlock, \& Hepburn, 2018). No entanto, o tratamento com vitamina D demonstrou ser eficaz na melhoria de certos sintomas da FB(Martins et al., 2020),(de Carvalho et al., 2018) como a percepção da dor e a hipersensibilidade musculo esquelética e, ainda, existem evidências que em pacientes com FB com redução dos níveis séricos de vitamina D (20.1 to $29 \mathrm{ng} / \mathrm{mL}$ como insuficiência e $<20 \mathrm{ng} / \mathrm{mL}$ como deficiência), uma combinação de suplementos desta vitamina e um antidepressivo convencional, como a trazodona, pode melhorar significativamente os sintomas físicos e psicológicos dos pacientes afetados(Mirzaei et al., 2018).

\section{Probióticos}

O sistema gastrointestinal parece ser afetado em pacientes com FB levando a quadros que podem incluir hipersensibilidade a componentes alimentares, doença celíaca, sensibilidade não-celíaca ao glúten, intolerância à lactose e FODMAPs (oligo-, di-, monossacáridos e poliois fermentáveis), assim como um aumento da imunoglobulina A secretora (sIgA) que está relacionada à fadiga, depressão maior e sintomas gastrointestinais (padrões intestinais irregulares e alternância entre diarreia e constipação)(P Roman et al., 2018).

Dado o fato da microbiota intestinal desempenhar um papel no neurodesenvolvimento de redes cerebrais relacionadas à regulação da emoção, esta pode ser um alvo potencial para a prevenção de ansiedade e transtornos depressivos em populações de risco (Slyepchenko, Carvalho, Cha, Kasper, \& McIntyre, 2014).

Embora os estudos sejam recentes, o uso de probióticos na FB tem sido preconizado. 
Uma preparação probiótica é um produto formado por microrganismos vivos que afeta beneficamente a saúde do organismo hospedeiro quando ingerido em dose adequada (Dinan \& Quigley, 2011).

Especificamente na FB o uso de um probiótico a partir de cepas bacterianas de Lactobacillus Rhamnosus, Casei, Acidophilus e Bifidobacterium Bifidus, (64 milhões de germes divididos em duas tomadas ao dia) pode melhorar a impulsividade e a capacidade de tomada de decisão nesses pacientes (Pablo Roman et al., 2018).

O efeito positivo dos probióticos na redução do comportamento impulsivo em pacientes com FB pode ser explicado em termos dos efeitos neuromodulatórios dos probióticos na serotonina e na dopamina através do nervo vago e do hipotálamo. No entanto, mais pesquisas são necessárias para explorar ainda mais os efeitos potenciais dos probióticos em outras funções cognitivas afetadas na FB, bem como em outras populações clínicas (Pablo Roman et al., 2018).

\section{Fitoterápicos}

O presente trabalho identificou um estudo utilizando uma combinação de fitoterápicos que apresentou resultados estatisticamente significantes na melhora dos sintomas da FB (Barmaki et al., 2019).

O referido estudo utilizou uma formulação para a utilização diurna e uma formulação noturna. A formulação diurna utilizada pela manhã era constituída por extratos de gengibre $50 \mathrm{mg}$, acerola $240 \mathrm{mg}$, vitamina C $120 \mathrm{mg}$, Spiraea Ulmaria 40 mg e geleia real $40 \mathrm{mg}$, já a formulação noturna tinha como constituintes passiflora $80 \mathrm{mg}$, camomila $80 \mathrm{mg}$, Spiraea Ulmaria $40 \mathrm{mg}$, Elymus repens $100 \mathrm{mg}$ e L-tirosina $45 \mathrm{mg}$.

Os resultados desta combinação mostraram que houve melhora nos escores psíquicos evidenciados na qualidade do sono, fadiga e ansiedade os quais são provavelmente relacionados à combinação de camomila e passiflora, que demonstraram propriedades ansiolíticas e hipnóticas em humanos e animais. Elymus repens, que contém 5- hidroxitriptofano (5-HT), um precursor da serotonina, também demonstrou aumentar as concentrações plasmáticas de melatonina, sugerindo que o aumento da disponibilidade de 5-HT em humanos pode afetar a síntese de melatonina e consequentemente a qualidade do sono. A Ltirosina, precursora da dopamina, melhora as facetas do controle cognitivo em situações com altas demandas cognitivas. Além de melhorar o sono, as propriedades tônicas da geleia real, acerola e vitamina $\mathrm{C}$ exercem um efeito adicional benéfico na fadiga. A vitamina C e E contidas na formulação contribuíram para a diminuição do estresse oxidativo (Barmaki et al., 2019).

O Crocus Sativus também foi um suplemento fitoterápico que apresentou resultados positivos no tratamento da FB. O estudo de Shakiba et al. (2018) (Shakiba et al., 2018) realizou uma comparação entre o uso do açafrão (Crocus Sativus) e a duloxetina a qual tem função de controle da dor e dos sintomas depressivos da FB com efeitos mínimos na fadiga e nos distúrbios do sono por meio da inibição seletiva da recaptação de serotonina e noradrenalina. Os participantes destes estudos foram randomicamente avaliados e alocados em dois grupos, os quais receberam $15 \mathrm{mg}$ de Crocus Sativus ou $30 \mathrm{mg}$ de duloxetina iniciando com uma capsula ao dia na primeira semana e duas cápsulas ao dia após a segunda semana numa duração total de 8 semanas. Ao final do estudo os efeitos do uso do Crocus Sativus foi similar àqueles da duloxetina. $\mathrm{O}$ uso do Crocus Sativus como coadjuvante no tratamento de sintomas depressivos vem se firmando como terapêutica eficaz no manejo dos pacientes portadores destas disfunções e pode ser comparável a substâncias tradicionais utilizadas no tratamento de ansiedade e relacionados ao humor como a fluoxetina por exemplo (Mazidi et al., 2016).

Outro suplemento utilizado que se destacou nesta revisão foi o cogumelo Ganoderma lucidum, que em chinês denomina-se Língzhī que significa "erva de potência espiritual", ou "cogumelo da imortalidade"(Arora, 1986). Destacamos um trabalho que concluiu que $6 \mathrm{~g} /$ dia de Ganoderma lucidum por 6 semanas melhoraram a aptidão física de mulheres que sofrem de FB. Especificamente, observou-se melhorias na resistência aeróbica, velocidade de caminhada e flexibilidade de membros inferiores. Dada a falta de estudos sobre o potencial mecanismo do Ganoderma lucidum na aptidão física, a melhor explicação baseou-se em estudos anteriores sobre o efeito antioxidante deste suplemento (Collado Mateo et al., 2015). 


\section{Conclusão}

A suplementação nutracêutica vem se constituindo uma alternativa válida para o tratamento da FB que pode contribuir com a melhora do quadro de dor e suas repercussões psíquicas e comportamentais.

O presente trabalho pôde destacar, que embora existam diversos trabalhos buscando evidenciar substâncias e produtos que possam ser benéficos para o tratamento da FB poucos atendem critérios de rigidez metodológica e efetividade clínica.

As substâncias como a CoQ10, vitamina D, probióticos e alguns fitoterápicos destacaram-se como elementos que, juntamente com as terapias tradicionais, atividade física e abordagens corpo-mente podem contribuir com a melhora da qualidade de vida dos portadores de FB.

Sugere-se estudos posteriores, diante da vasta gama de princípios ativos, principalmente com fitoterápicos e nutracêuticos de origem brasileira que ainda não tenham sido pesquisados dentro do rigor científico para a utilização na FB disfunção esta cada vez mais presente nos dias atuais.

\section{Referências}

Arora, D. (1986). Mushrooms Demystified: A Comprehensive Guide to the Fleshy Fungi. Ten Speed Press.

Barmaki, M., Maindet-Dominici, C., Nizard, J., Baron, D., Russ, I., Fardellone, P., ... Bertin, P. (2019). Multicenter, Prospective, Controlled Double-Blind Study Comparing Fib-19-01, A Phytotherapy Treatment, To A Dietary Supplement And To Conventional Care In Patients Suffering From Fibromyalgia. Alternative Therapies in Health and Medicine, 25(4), 46-53.

Binkiewicz-Glinska, A., Bakula, S., Tomczak, H., Landowski, J., Ruckemann-Dziurdzinska, K., Zaborowska-Sapeta, K., \& Kiebzak, W. (2015). Fibromyalgia Syndrome - a multidisciplinary approach. Psychiatria Polska, 49(4), 801-810. https://doi.org/10.12740/psychiatriapolska.pl/online-first/4

Collado Mateo, D., Pazzi, F., Dominguez Munoz, F. J., Martin Martinez, J. P., Olivares, P. R., Gusi, N., \& Adsuar, J. C. (2015). Ganoderma Lucidum Improves Physical Fitness In Women With Fibromyalgia. Nutricion Hospitalaria, 32(5), 2126-2135. https://doi.org/10.3 305/nh.2015.32.5.9601

Cordero, M. D., Alcocer-Gómez, E., de Miguel, M., Cano-García, F. J., Luque, C. M., Fernández-Riejo, P., \& Sánchez-Alcazar, J. A. (2011). Coenzyme $\mathrm{Q}(10)$ : a novel therapeutic approach for Fibromyalgia? case series with 5 patients. Mitochondrion, 11(4), 623-625. https://doi.org/10.1016/j.mito.2011.03.122

Cordero, M. D., de Miguel, M., Carmona-López, I., Bonal, P., Campa, F., \& Moreno-Fernández, A. M. (2010). Oxidative stress and mitochondrial dysfunction in fibromyalgia. Neuro Endocrinology Letters, 31(2), 169-173.

de Carvalho, J. F., da Rocha Araujo, F. A. G., da Mota, L. M. A., Aires, R. B., \& de Araujo, R. P. (2018). Vitamin D Supplementation Seems to Improve Fibromyalgia Symptoms: Preliminary Results. The Israel Medical Association Journal : IMAJ, 20(6), 379-381.

Di Pierro, F., Rossi, A., Consensi, A., Giacomelli, C., \& Bazzichi, L. (2017). Role for a water-soluble form of CoQ10 in female subjects affected by fibromyalgia. A preliminary study. Clinical and Experimental Rheumatology, 35 Suppl 1(3), 20-27.

Dinan, T. G., \& Quigley, E. M. (2011, December). Probiotics in the treatment of depression: science or science fiction? The Australian and New Zealand Journal of Psychiatry. England. https://doi.org/10.3109/00048674.2011.613766

Ellis, S. D., Kelly, S. T., Shurlock, J. H., \& Hepburn, A. L. N. (2018). The role of vitamin D testing and replacement in fibromyalgia: a systematic literature review. BMC Rheumatology, 2, 28. https://doi.org/10.1186/s41927-018-0035-6

Häuser, W., Ablin, J., Fitzcharles, M.-A., Littlejohn, G., Luciano, J. V, Usui, C., \& Walitt, B. (2015). Fibromyalgia. Nature Reviews. Disease Primers, 1, 15022. https://doi.org/10.1038/nrdp.2015.22

Hauser, W., Sarzi-Puttini, P., \& Fitzcharles, M.-A. (2019). Fibromyalgia syndrome: under-, over- and misdiagnosis. Clinical and Experimental Rheumatology, 37 Suppl 1(1), 90-97.

Jorat, M. V., Tabrizi, R., Kolahdooz, F., Akbari, M., Salami, M., Heydari, S. T., \& Asemi, Z. (2019, April 1). The effects of coenzyme Q10 supplementation on biomarkers of inflammation and oxidative stress in among coronary artery disease: a systematic review and meta-analysis of randomized controlled trials. Inflammopharmacology. Birkhauser Verlag AG. https://doi.org/10.1007/s10787-019-00572-x

Liberati, A., Altman, D. G., Tetzlaff, J., Mulrow, C., Gøtzsche, P. C., Ioannidis, J. P. A., \& Moher, D. (2009). The PRISMA statement for reporting systematic reviews and meta-analyses of studies that evaluate health care interventions: explanation and elaboration. PLoS Medicine, 6(7), e1000100. https://doi.org/10.1371/journal.pmed.1000100

Martins, Y. A., Cardinali, C. A. E. F., Ravanelli, M. I., \& Brunaldi, K. (2020). Is hypovitaminosis D associated with fibromyalgia? A systematic review. Nutrition Reviews, 78(2), 115-133. https://doi.org/10.1093/nutrit/nuz033

Mazidi, M., Shemshian, M., Mousavi, S. H., Norouzy, A., Kermani, T., Moghiman, T., \& Ferns, G. A. A. (2016). A double-blind, randomized and placebocontrolled trial of Saffron (Crocus sativus L.) in the treatment of anxiety and depression. Journal of Complementary \& Integrative Medicine, 13(2), 195-199. https://doi.org/10.1515/jcim-2015-0043 
Mirzaei, A., Zabihiyeganeh, M., Jahed, S. A., Khiabani, E., Nojomi, M., \& Ghaffari, S. (2018). Effects of vitamin D optimization on quality of life of patients with fibromyalgia: A randomized controlled trial. Medical Journal of the Islamic Republic of Iran, 32, 29. https://doi.org/10.14196/mjiri.32.29

Mizobuti, D. S., Fogaça, A. R., Moraes, F. dos S. R., Moraes, L. H. R., Mâncio, R. D., Hermes, T. de A., \& Minatel, E. (2019). Coenzyme Q10 supplementation acts as antioxidant on dystrophic muscle cells. Cell Stress and Chaperones, 24(6), 1175-1185. https://doi.org/10.1007/s12192-019-01039-2

Mousavinejad, E., Ghaffari, M. A., Riahi, F., Hajmohammadi, M., Tiznobeyk, Z., \& Mousavinejad, M. (2018). Coenzyme Q10 supplementation reduces oxidative stress and decreases antioxidant enzyme activity in children with autism spectrum disorders. Psychiatry Research, 265, 62-69. https://doi.org/10.1016/j.psychres.2018.03.061

Pereira A.S. et al. (2018). Metodologia da pesquisa científica. UFSM. https://repositorio.ufsm.br/bitstream/handle/1/15824/Lic_Computacao_MetodologiaPesquisa-Cientifica.pdf?

Ramezani, M., Sahraei, Z., Simani, L., Heydari, K., \& Shahidi, F. (2018). Coenzyme Q10 supplementation in acute ischemic stroke: Is it beneficial in shortterm administration? Nutritional Neuroscience. https://doi.org/10.1080/1028415X.2018.1541269

Roman, P, Carrillo-Trabalón, F., Sánchez-Labraca, N., Cañadas, F., Estévez, A. F., \& Cardona, D. (2018). Are probiotic treatments useful on fibromyalgia syndrome or chronic fatigue syndrome patients? A systematic review. Beneficial Microbes, 9(4), 603-611. https://doi.org/10.3920/BM2017.0125

Roman, Pablo, Estevez, A. F., Miras, A., Sanchez-Labraca, N., Canadas, F., Vivas, A. B., \& Cardona, D. (2018). A Pilot Randomized Controlled Trial to Explore Cognitive and Emotional Effects of Probiotics in Fibromyalgia. Scientific Reports, 8(1), 10965. https://doi.org/10.1038/s41598-018-29388-5

Sahota, O. (2014). Understanding vitamin D deficiency. Age and Ageing, 43(5), 589-591. https://doi.org/10.1093/ageing/afu104

Sawaddiruk, P., Apaijai, N., Paiboonworachat, S., Kaewchur, T., Kasitanon, N., Jaiwongkam, T., \& Chattipakorn, S. C. (2019). Coenzyme Q10 supplementation alleviates pain in pregabalin-treated fibromyalgia patients via reducing brain activity and mitochondrial dysfunction. Free Radical Research, 53(8), 901-909. https://doi.org/10.1080/10715762.2019.1645955

Shakiba, M., Moazen-Zadeh, E., Noorbala, A. A., Jafarinia, M., Divsalar, P., Kashani, L., \& Akhondzadeh, S. (2018). Saffron (Crocus sativus) versus duloxetine for treatment of patients with fibromyalgia: A randomized double-blind clinical trial. Avicenna Journal of Phytomedicine, 8(6), 513-523. Retrieved from https://pubmed.ncbi.nlm.nih.gov/30456199

Slyepchenko, A., Carvalho, A. F., Cha, D. S., Kasper, S., \& McIntyre, R. S. (2014). Gut emotions - mechanisms of action of probiotics as novel therapeutic targets for depression and anxiety disorders. CNS \& Neurological Disorders Drug Targets, 13(10), 1770-1786. https://doi.org/10.2174/1871527313666141130205242

Stewart, J. A., Mailler-Burch, S., Müller, D., Studer, M., von Känel, R., Grosse Holtforth, M., ... Egloff, N. (2019). Rethinking the criteria for fibromyalgia in 2019: the ABC indicators. Journal of Pain Research, 12, 2115-2124. https://doi.org/10.2147/JPR.S205299

Theoharides, T. C., Tsilioni, I., Arbetman, L., Panagiotidou, S., Stewart, J. M., Gleason, R. M., \& Russell, I. J. (2015). Fibromyalgia syndrome in need of effective treatments. The Journal of Pharmacology and Experimental Therapeutics, 355(2), 255-263. https://doi.org/10.1124/jpet.115.227298

Wolfe, F., Clauw, D. J., Fitzcharles, M.-A., Goldenberg, D. L., Katz, R. S., Mease, P., \& Yunus, M. B. (2010). The American College of Rheumatology preliminary diagnostic criteria for fibromyalgia and measurement of symptom severity. Arthritis Care \& Research, 62(5), 600-610. https://doi.org/10.1002/acr.20140 